\title{
N-C Bond Formation Promoted by a Hafnocene Dinitrogen Complex: Comparison of Zirconium and Hafnium Congeners.
}

Wesley H. Bernskoetter, Andrea V. Olmos, Jamie A. Pool, Emil Lobkovsky, Paul J. Chirik*

Baker Laboratory, Department of Chemistry and Chemical Biology,

Cornell University, Ithaca, NY 14853

-Supporting Information- 
Table of Contents

$\begin{array}{ll}\text { Experimental Procedures } & \text { S3 }\end{array}$

$\begin{array}{ll}\text { References } & \text { S8 }\end{array}$ 


\section{Experimental Procedures}

General Considerations. All air- and moisture-sensitive manipulations were carried out using standard high vacuum line, Schlenk or cannula techniques or in an M. Braun inert atmosphere drybox containing an atmosphere of purified nitrogen. The M. Braun drybox was equipped with a cold well designed for freezing samples in liquid nitrogen. Solvents for air- and moisture-sensitive manipulations were dried and deoxygenated using literature procedures. ${ }^{1}$ Toluene, benzene, pentane and heptane were further dried by distillation from "titanocene". ${ }^{2}$ Deuterated solvents for NMR spectroscopy were distilled from sodium metal under an atmosphere of argon and stored over $4 \AA$ molecular sieves. Argon and hydrogen gas were purchased from Airgas Incorporated and passed through a column containing manganese oxide on vermiculite and $4 \AA$ molecular sieves before admission to the high vacuum line. ${ }^{1} \mathrm{H}$ NMR spectra were recorded on a Varian Inova 400 Spectrometer operating at $399.860 \mathrm{MHz}$. All chemical shifts are reported relative to $\mathrm{SiMe}_{4}$ using ${ }^{1} \mathrm{H}$ (residual) chemical shifts of the solvent as a secondary standard. ${ }^{2} \mathrm{H},{ }^{13} \mathrm{C}$,

${ }^{31} \mathrm{P}$, and ${ }^{15} \mathrm{~N}$ NMR spectra were recorded on a Varian Inova 500 Spectrometer operating at $76.848,125.716,161.83$, and $50.663 \mathrm{MHz}$, respectively. ${ }^{2} \mathrm{H}$ and ${ }^{13} \mathrm{C}$ chemical shifts are reported relative to $\mathrm{SiMe}_{4}$ using chemical shifts of the solvent as a secondary standard. ${ }^{31} \mathrm{P}$ and ${ }^{15} \mathrm{~N}$ chemical shifts are reported relative $\mathrm{H}_{3} \mathrm{PO}_{4}$ and liquid $\mathrm{NH}_{3}$, respectively, using external standard standards. The compounds $\mathbf{1}-\mathbf{N}_{\mathbf{2}}{ }^{3}{ }^{2}-\mathbf{N}_{\mathbf{2}},{ }^{4}$ and $\left(\eta^{5}-\mathrm{C}_{5} \mathrm{Me}_{4} \mathrm{H}\right)_{2} \mathrm{ZrH}_{2}{ }^{5}$ were prepared according to literature procedures. Phenyl isocyanate, trimethylphosphine, and tert-butyl acetylene were purchased from Acros or Aldrich and dried over $4 \AA$ molecular sieves prior to use. 
Single crystals suitable for X-ray diffraction were coated with polyisobutylene oil in a drybox, transferred to a nylon loop and then quickly transferred to the goniometer head of a Bruker X8 APEX2 diffractometer equipped with a molybdenum X-ray tube (_ $=0.71073 \AA$ A). Preliminary data revealed the crystal system. A hemisphere routine was used for data collection and determination of lattice constants. The space group was identified and the data were processed using the Bruker SAINT+ program and corrected for absorption using SADABS. The structures were solved using direct methods (SHELXS) completed by subsequent Fourier synthesis and refined by full-matrix least squares procedures. Infrared spectroscopy was conducted on a Mattson RS-10500 Research Series FT-IR spectrometer calibrated with a polystyrene standard. Elemental analyses were performed at Robertson Microlit Laboratories, Inc., in Madison, NJ.

Characterization of $\left[\left(\eta^{5}-\mathrm{C}_{5} \mathbf{M e}_{4} \mathrm{H}\right)_{2} \mathrm{Zr}\left(\mathbf{P M e}_{3}\right)\right]_{2}\left(\mu_{2}, \eta^{1}, \eta^{1}-\mathbf{N}_{2}\right)$ (1-PMe $)$. A J. Young NMR tube was charged with $0.035 \mathrm{~g}(0.050 \mathrm{mmol})$ of $\mathbf{1}-\mathbf{N}_{2}$ and approximately $0.5 \mathrm{~mL}$ of benzene- $d_{6}$. On the vacuum line, the tube was submerged in liquid nitrogen and degassed. While frozen, 2 equivalents (59 torr in a $31.6 \mathrm{~mL}$ bulb) of $\mathrm{PMe}_{3}$ were added to the tube. The tube was sealed and warmed to ambient temperature forming deep red solution. Monitoring the reaction by ${ }^{1} \mathrm{H}$ NMR spectroscopy revealed complete conversion after two hours. Attempts to isolate the product by exposure to vacuum resulted in irreversible decomposition. ${ }^{1} \mathrm{H}$ NMR (benzene- $d_{6}, 23{ }^{\circ} \mathrm{C}$ ): $\delta=1.14$ (bs, $18 \mathrm{H}, \mathrm{P} M e_{3}$ ), 2.06 (bs, $24 \mathrm{H}$, $\mathrm{C}_{5} M e_{4} \mathrm{H}$ ), 2.14 (bs, $24 \mathrm{H}, \mathrm{C}_{5} M e_{4} \mathrm{H}$ ), 4.99 (bs, $4 \mathrm{H}, \mathrm{C}_{5} \mathrm{Me}_{4} H$ ). ${ }^{1} \mathrm{H}$ NMR (toluene- $d_{8},-70{ }^{\circ} \mathrm{C}$ ): $\delta=1.04\left(\mathrm{bs}, 18 \mathrm{H}, \mathrm{PMe}_{3}\right), 1.83\left(\mathrm{~s} 6 \mathrm{H}, \mathrm{C}_{5} M e_{4} \mathrm{H}\right), 1.90$ (bs, 6H, $\left.\mathrm{C}_{5} M e_{4} \mathrm{H}\right), 1.98$ (bs, 6H, $\mathrm{C}_{5} M e_{4} \mathrm{H}$ ), $2.02\left(\mathrm{~s}, 6 \mathrm{H}, \mathrm{C}_{5} M e_{4} \mathrm{H}\right), 2.07$ (bs, 6H, $\left.\mathrm{C}_{5} M e_{4} \mathrm{H}\right), 2.12$ (bs, 6H, $\left.\mathrm{C}_{5} M e_{4} \mathrm{H}\right), 2.22(\mathrm{~s}$, 
$6 \mathrm{H}, \mathrm{C}_{5} M e_{4} \mathrm{H}$ ), $2.62\left(\mathrm{bs}, 6 \mathrm{H}, \mathrm{C}_{5} M e_{4} \mathrm{H}\right), 4.74$ (bs, $2 \mathrm{H}, \mathrm{C}_{5} \mathrm{Me}_{4} H$ ), 4.98 (bs, $2 \mathrm{H}, \mathrm{C}_{5} \mathrm{Me}_{4} H$ ). ${ }^{13} \mathrm{C}$ NMR (benzene- $\left.d_{6}, 23^{\circ} \mathrm{C}\right): \delta=13.70\left(\mathrm{PMe} e_{3}\right), 16.81,19.59(\mathrm{CpMe}), 104.50,105.60(\mathrm{Cp})$. ${ }^{31} \mathrm{P}$ NMR (benzene- $d_{6}, 23{ }^{\circ} \mathrm{C}$ ): $\delta=-7.26$ (bs). ${ }^{15} \mathrm{~N}$ NMR (benzene- $d_{6}, 23{ }^{\circ} \mathrm{C}$ ): $\delta=533.6$ (bs).

Hydrogenation of 1-PMe 3 . A J. Young NMR tube was charged with $0.020 \mathrm{~g}(0.023$ mmol) 1-PMe $\mathbf{P}_{3}$ and approximately $0.5 \mathrm{~mL}$ benzene- $d_{6}$. On the vacuum line, the tube was submerged in liquid nitrogen and degassed, after which 1 atmosphere of dihydrogen was admitted. The tube was warmed to ambient temperature resulting in a color change from deep red to pale orange. The organometallic product was identified as $\left(\eta^{5}\right.$ $\left.\mathrm{C}_{5} \mathrm{Me}_{4} \mathrm{H}\right)_{2} \mathrm{Zr}\left(\mathrm{PMe}_{3}\right) \mathrm{H}_{2}$ by comparison to an authentic sample.

Preparation of $\left(\eta^{5}-\mathrm{C}_{5} \mathrm{Me}_{4} \mathrm{H}\right)_{2} \mathrm{Zr}\left(\mathrm{PMe}_{3}\right) \mathrm{H}_{2}$. A J. Young NMR tube was charged with $0.017 \mathrm{~g}(0.049 \mathrm{mmol})$ of $\left(\eta^{5}-\mathrm{C}_{5} \mathrm{Me}_{4} \mathrm{H}\right)_{2} \mathrm{ZrH}_{2}$ and approximately $0.5 \mathrm{~mL}$ benzene- $d_{6}$. On the vacuum line, the tube was submerged in liquid nitrogen and degassed, after which 1 equivalent of $\mathrm{PMe}_{3}$ was admitted. The tube was warmed to ambient temperature, and the reaction monitored by NMR spectroscopy. ${ }^{1} \mathrm{H}$ NMR (benzene- $\left.d_{6}, 23{ }^{\circ} \mathrm{C}\right): \delta=1.06(\mathrm{~d}, 4.8$ $\left.\mathrm{Hz}, \mathrm{P} M e_{3}\right), 1.38$ (bs, 2H, $\left.\mathrm{Zr}-H\right) 1.96\left(\mathrm{~s}, 12 \mathrm{H}, \mathrm{C}_{5} M e_{4} \mathrm{H}\right), 2.24\left(\mathrm{~s}, 12 \mathrm{H}, \mathrm{C}_{5} M e_{4} \mathrm{H}\right), 4.79(\mathrm{~s}$, $\left.2 \mathrm{H}, \mathrm{C}_{5} \mathrm{Me}_{4} H\right) .{ }^{13} \mathrm{C}$ NMR (benzene- $\left.\left.d_{6}, 23{ }^{\circ} \mathrm{C}\right): \delta=12.84,15.61(\mathrm{CpMe}), 22.10(\mathrm{PMe})_{3}\right)$, 103.95, 111.87, $113.71(\mathrm{Cp}) .{ }^{31} \mathrm{P}$ NMR (benzene- $d_{6}, 23{ }^{\circ} \mathrm{C}$ ): $\delta=-14.16$ (bs).

Preparation of 3. A $20 \mathrm{~mL}$ scintillation vial was charged with $0.103 \mathrm{~g}(0.114 \mathrm{mmol})$ of 2- $\mathbf{N}_{2}$ and approximately $10 \mathrm{~mL}$ toluene. The resulting dark purple solution was chilled to 
$-30{ }^{\circ} \mathrm{C}$ and $0.025(.210 \mathrm{mmol})$ of phenyl isocyanate was added with stirring. The reaction mixture was warmed to ambient temperature and stirred for 2 hours. Over this course of the two hours, the solution changed color to orange. The solvent was removed in vacuo and the residue was recrystallized from approximately $5 \mathrm{~mL}$ of diethyl ether to afford $0.058 \mathrm{~g}(46 \%)$ of 3 as orange crystals. Anal. Calcd for $\mathrm{C}_{50} \mathrm{H}_{62} \mathrm{Hf}_{2} \mathrm{~N}_{4} \mathrm{O}_{2}$ : C, 54.20; $\mathrm{H}, 5.64$; $\mathrm{N}, 5.06$. Found: C, 53.87; H, 6.02; N, 4.68. ${ }^{1} \mathrm{H}$ NMR (benzene- $\left.d_{6}, 23{ }^{\circ} \mathrm{C}\right): \delta=1.78(\mathrm{bs}$, $\left.6 \mathrm{H}, \mathrm{C}_{5} M e_{4} \mathrm{H}\right), 1.83\left(\mathrm{bs}, 6 \mathrm{H}, \mathrm{C}_{5} M e_{4} \mathrm{H}\right), 1.85\left(\mathrm{bs}, 6 \mathrm{H}, \mathrm{C}_{5} M e_{4} \mathrm{H}\right), 1.94\left(\mathrm{~s}, 6 \mathrm{H}, \mathrm{C}_{5} M e_{4} \mathrm{H}\right), 1.95$ $\left(\mathrm{s}, 6 \mathrm{H}, \mathrm{C}_{5} M e_{4} \mathrm{H}\right), 1.96\left(\mathrm{bs}, 6 \mathrm{H}, \mathrm{C}_{5} M e_{4} \mathrm{H}\right), 2.04\left(\mathrm{~s}, 6 \mathrm{H}, \mathrm{C}_{5} M e_{4} \mathrm{H}\right), 2.18\left(\mathrm{~s}, 6 \mathrm{H}, \mathrm{C}_{5} M e_{4} \mathrm{H}\right)$, $5.93\left(\mathrm{bs}, 2 \mathrm{H}, \mathrm{C}_{5} \mathrm{Me}_{4} H\right), 6.25\left(\mathrm{bs}, 2 \mathrm{H}, \mathrm{C}_{5} \mathrm{Me}_{4} H\right), 6.95-7.92$ (PhNCO). ${ }^{1} \mathrm{H}$ NMR (toluene$\left.d_{8},-70{ }^{\circ} \mathrm{C}\right):{ }_{-} 1.48\left(\mathrm{~s}, 3 \mathrm{H}, \mathrm{C}_{5} M e_{4} \mathrm{H}\right), 1.53\left(\mathrm{bs}, 3 \mathrm{H}, \mathrm{C}_{5} M e_{4} \mathrm{H}\right), 1.59\left(\mathrm{bs}, 3 \mathrm{H}, \mathrm{C}_{5} M e_{4} \mathrm{H}\right), 1.64$ (bs, 3H, $\left.\mathrm{C}_{5} M e_{4} \mathrm{H}\right), 1.70\left(\mathrm{bs}, 3 \mathrm{H}, \mathrm{C}_{5} M e_{4} \mathrm{H}\right), 1.79\left(\mathrm{~s}, 3 \mathrm{H}, \mathrm{C}_{5} M e_{4} \mathrm{H}\right), 1.83\left(\mathrm{~s}, 3 \mathrm{H}, \mathrm{C}_{5} M e_{4} \mathrm{H}\right)$, $1.86\left(\mathrm{~s}, 3 \mathrm{H}, \mathrm{C}_{5} M e_{4} \mathrm{H}\right), 1.90\left(\mathrm{~s}, 6 \mathrm{H}, \mathrm{C}_{5} M e_{4} \mathrm{H}\right), 2.18\left(\mathrm{bs}, 3 \mathrm{H}, \mathrm{C}_{5} M e_{4} \mathrm{H}\right), 2.23$ (bs, 3H, $\left.\mathrm{C}_{5} M e_{4} \mathrm{H}\right), 2.26\left(\mathrm{bs}, 3 \mathrm{H}, \mathrm{C}_{5} M e_{4} \mathrm{H}\right), 5.44\left(\mathrm{~s}, 1 \mathrm{H}, \mathrm{C}_{5} \mathrm{Me}_{4} H\right), 5.55\left(\mathrm{~s}, 1 \mathrm{H}, \mathrm{C}_{5} \mathrm{Me}_{4} H\right), 6.21(\mathrm{bs}$, $\left.1 \mathrm{H}, \mathrm{C}_{5} \mathrm{Me}_{4} H\right), 6.32\left(\mathrm{~s}, 1 \mathrm{H}, \mathrm{C}_{5} \mathrm{Me}_{4} H\right), 6.98-8.17(P h \mathrm{NCO})$ (three $\mathrm{C}_{5} M e_{4} \mathrm{H}$ resonances not resolved). ${ }^{13} \mathrm{C}$ NMR (benzene- $\left.d_{6}, 23{ }^{\circ} \mathrm{C}\right): \delta=12.36,12.65,13.03,13.12,13.31,13.39$, 13.40, 13.47 (CpMe), 109.95, 111.61, 112.27, 121.52, 124.96, 126.04, 126.87, 127.47, $129.71,131.73,140.56,151.79,153.87(\mathrm{Cp} / \mathrm{PhNCO}) .{ }^{15} \mathrm{~N}$ NMR (benzene- $\left.d_{6}, 23{ }^{\circ} \mathrm{C}\right): \delta=$ $423.7(\mathrm{Hf}=N(1)), 181.5 \mathrm{ppm}(\mathrm{Hf}-N(2)-\mathrm{C}(37))$. IR $($ Benzene $): v=1619,1591 \mathrm{~cm}^{-1}$.

Preparation of 4. A $20 \mathrm{~mL}$ scintillation vial was charged with $0.078 \mathrm{~g}(0.090 \mathrm{mmol})$ of 2- $\mathbf{N}_{2}$ and $10 \mathrm{~mL}$ toluene. The solution was chilled to $-30{ }^{\circ} \mathrm{C}$ and $0.035 \mathrm{~g}(.296 \mathrm{mmol})$ of phenyl isocyanate was added with stirring. The reaction mixture was warmed to ambient temperature and stirred for 4 hours, at which time the solution had changed from dark 
purple to pale yellow. The solvent was removed in vacuo and the residue was recystallized from a $10 \mathrm{~mL}$ solution of toluene/pentane ( $1: 5)$ to afford $0.050 \mathrm{~g}(45 \%)$ of $\mathbf{4}$ as colorless crystals. Compound $\mathbf{4}$ can also be prepared directly from $\mathbf{3}$ by addition of one equivalent of phenyl isocyanate. Anal. Calcd for $\mathrm{C}_{57} \mathrm{H}_{67} \mathrm{Hf}_{2} \mathrm{~N}_{5} \mathrm{O}_{3}$ : C, 55.79; H, 5.50; $\mathrm{N}$, 5.71. Found: $\mathrm{C}, 55.53 ; \mathrm{H}, 5.87 ; \mathrm{N}, 5.76 .{ }^{1} \mathrm{H}$ NMR (benzene- $d_{6}, 23{ }^{\circ} \mathrm{C}$ ): $\delta=1.76$ (s, $\left.6 \mathrm{H}, \mathrm{C}_{5} M e_{4} \mathrm{H}\right), 1.79\left(\mathrm{~s}, 6 \mathrm{H}, \mathrm{C}_{5} M e_{4} \mathrm{H}\right), 1.81\left(\mathrm{~s}, 6 \mathrm{H}, \mathrm{C}_{5} M e_{4} \mathrm{H}\right), 1.89\left(\mathrm{~s}, 12 \mathrm{H}, \mathrm{C}_{5} M e_{4} \mathrm{H}\right), 1.90$ (s, 6H, $\left.\mathrm{C}_{5} M e_{4} \mathrm{H}\right), 2.19\left(\mathrm{~s}, 6 \mathrm{H}, \mathrm{C}_{5} M e_{4} \mathrm{H}\right), 2.20\left(\mathrm{~s}, 6 \mathrm{H}, \mathrm{C}_{5} M e_{4} \mathrm{H}\right), 5.59\left(\mathrm{~s}, 2 \mathrm{H}, \mathrm{C}_{5} \mathrm{Me}_{4} H\right), 5.60$ (s, $2 \mathrm{H}, \mathrm{C}_{5} \mathrm{Me}_{4} H$ ), 6.92- $7.72(\mathrm{PhNCO}) .{ }^{13} \mathrm{C}$ NMR (benzene- $\left.d_{6}, 23{ }^{\circ} \mathrm{C}\right): \delta=11.92,12.20$, 12.57, 12.79, 13.11, 13.29, 14.88, 15.17 (CpMe), 109.11, 109.80, 118.51, 119.94, 121.27, $122.13,123.07,123.15,124.11,124.57,127.14,127.76,128.96,130.88,142.37,149.95$, 150.72, 151.07, 163.33, $164.99(\mathrm{Cp} / \mathrm{PhNCO}) .{ }^{15} \mathrm{~N}$ NMR (benzene- $\left.d_{6}, 23{ }^{\circ} \mathrm{C}\right): \delta=173.8$ $(\mathrm{d}, 6.6 \mathrm{~Hz}) 187.1(\mathrm{~d}, 6.6 \mathrm{~Hz})$. IR (Benzene): $v=1625,1500 \mathrm{~cm}^{-1}$.

Characerization of 5. A J. Young NMR tube was charged with $0.005 \mathrm{~g}(.005 \mathrm{mmol})$ of 3 and approximately $0.5 \mathrm{~mL}$ of benzene- $d_{6}$. Then $0.010 \mathrm{~g}$ of a stock solution of $p$-tolyl isocyanate $\left(29 \mathrm{mg} / 432 \mathrm{mg}\right.$ benzene- $\left.d_{6}\right)$ was added. The solution immediately turned from orange to colorless. The reaction was monitored by NMR spectroscopy. ${ }^{1} \mathrm{H}$ NMR (benzene- $\left.d_{6}, 23{ }^{\circ} \mathrm{C}\right): \delta=1.77\left(\mathrm{~s}, 6 \mathrm{H}, \mathrm{C}_{5} M e_{4} \mathrm{H}\right), 1.81\left(\mathrm{~s}, 6 \mathrm{H}, \mathrm{C}_{5} M e_{4} \mathrm{H}\right), 1.82(\mathrm{~s}, 6 \mathrm{H}$, $\left.\mathrm{C}_{5} M e_{4} \mathrm{H}\right), 1.89\left(M e \mathrm{C}_{6} \mathrm{H}_{4}\right) 1.90\left(\mathrm{~s}, 6 \mathrm{H}, \mathrm{C}_{5} M e_{4} \mathrm{H}\right), 1.91\left(\mathrm{~s}, 6 \mathrm{H}, \mathrm{C}_{5} M e_{4} \mathrm{H}\right), 1.92(\mathrm{~s}, 6 \mathrm{H}$, $\left.\mathrm{C}_{5} \mathrm{Me}_{4} \mathrm{H}\right), 2.21\left(\mathrm{~s}, 12 \mathrm{H}, \mathrm{C}_{5} \mathrm{Me}_{4} \mathrm{H}\right)$ 1. $5.33\left(\mathrm{~s}, 2 \mathrm{H}, \mathrm{C}_{5} \mathrm{Me}_{4} H\right), 5.78$ (s, $\left.2 \mathrm{H}, \mathrm{C}_{5} \mathrm{Me}_{4} H\right), 6.96-$ 7.60 (ArNCO). ${ }^{13} \mathrm{C}$ NMR (benzene- $d_{6}, 23{ }^{\circ} \mathrm{C}$ ): $\delta=11.90,12.21,12.59,12.75,13.12$, 13.34, 14.91, 15.22 (CpMe), 21.24 (p-tolyl), 109.06, 109.92, 118.45, 119.57, 121.21, $121.93,123.12,124.14,124.58,127.11,128.89,128.97,130.87,132.23,135.64,142.40$, 
$147.25,150.71,151.12,163.31,165.10(\mathrm{Cp} / \mathrm{ArNCO}) .{ }^{15} \mathrm{~N}$ NMR (benzene- $\left.d_{6}\right): \delta=206.1$ $\left(\mathrm{d}, J_{N-N}=9.5 \mathrm{~Hz}, N-\mathrm{C}\right) ; 194.8\left(\mathrm{~d}, J_{N-N}=9.5 \mathrm{~Hz}, N-\mathrm{H}\right)$.

Preparation of 6. A $25 \mathrm{~mL}$ round-bottomed flask equipped with a $180^{\circ}$ needle valve was charged with $0.058 \mathrm{~g}(0.052 \mathrm{mmol})$ of $\mathbf{3}$ and approximately $10 \mathrm{~mL}$ of toluene. On the vacuum line, the flask was submerged in liquid nitrogen and evacuated. One equivalent (31 torr in a $31.6 \mathrm{~mL}$ bulb) of tert-butyl acetylene was added and the reaction mixture warmed to ambient temperature and stirred for one hour. Over this time a yellow solution formed. The solvent was removed in vacuo and the resulting residue extracted with cold pentane. The solvent was again removed yielding $0.049 \mathrm{~g}$ (79\%) of 5 as a yellow-brown solid. Anal. Calcd for $\mathrm{C}_{56} \mathrm{H}_{72} \mathrm{Hf}_{2} \mathrm{~N}_{4} \mathrm{O}_{2}$ : C, 56.51; H, 6.10; N, 4.71. Found: C, 56.59; H, 6.02; N, 4.43. ${ }^{1} \mathrm{H}$ NMR (benzene- $\left.d_{6}, 23{ }^{\circ} \mathrm{C}\right): \delta=1.39\left(\mathrm{~s}, 9 \mathrm{H}, \mathrm{C}_{-} \mathrm{C}^{t} B u\right), 1.83(\mathrm{~s}, 6 \mathrm{H}$, $\left.\mathrm{C}_{5} M e_{4} \mathrm{H}\right), 1.88\left(\mathrm{~s}, 6 \mathrm{H}, \mathrm{C}_{5} M e_{4} \mathrm{H}\right), 1.89\left(\mathrm{~s}, 6 \mathrm{H}, \mathrm{C}_{5} M e_{4} \mathrm{H}\right), 1.94\left(\mathrm{~s}, 6 \mathrm{H}, \mathrm{C}_{5} M e_{4} \mathrm{H}\right), 1.95(\mathrm{~s}, 6 \mathrm{H}$, $\left.\mathrm{C}_{5} M e_{4} \mathrm{H}\right), 2.08\left(\mathrm{~s}, 6 \mathrm{H}, \mathrm{C}_{5} M e_{4} \mathrm{H}\right), 2.10\left(\mathrm{~s}, 6 \mathrm{H}, \mathrm{C}_{5} M e_{4} \mathrm{H}\right), 2.12\left(\mathrm{~s}, 6 \mathrm{H}, \mathrm{C}_{5} M e_{4} \mathrm{H}\right), 2.43(\mathrm{~N}-\mathrm{H})$, $5.33\left(\mathrm{~s}, 2 \mathrm{H}, \mathrm{C}_{5} \mathrm{Me}_{4} H\right), 5.78\left(\mathrm{~s}, 2 \mathrm{H}, \mathrm{C}_{5} \mathrm{Me}_{4} H\right), 6.95-7.60(P h \mathrm{NCO}) .{ }^{13} \mathrm{C}$ NMR (benzene- $d_{6}$, $\left.23{ }^{\circ} \mathrm{C}\right): \delta=11.43,12.63,12.65,13.03,13.72,13.93,14.54,14.83(\mathrm{CpMe}), 34.76\left(\mathrm{CMe}_{3}\right)$, 109.52, 110.15, 112.19, 117.76, 121.63, 122.61, 125.00, 131.06, 139.58, 140.07, 142.07, $151.47(\mathrm{Cp} / \mathrm{PhNCO}) .{ }^{15} \mathrm{~N}$ NMR (benzene- $\left.d_{6}, 23{ }^{\circ} \mathrm{C}\right): \delta=190.8 \mathrm{ppm}$. IR $(\mathrm{KBr}): v_{\mathrm{N}-\mathrm{H}}=$ $3290 ; v_{\mathrm{C}=\mathrm{C}}=2220 \mathrm{~cm}^{-1}$.

\section{References}

${ }^{1}$ Pangborn, A.B.; Giardello, M.A.; Grubbs, R.H.; Rosen, R.K.; Timmers, F.J. Organometallics 1996, 15, 1518. 
${ }^{2}$ Marvich, R.H.; Brintzinger, H.H. J. Am. Chem. Soc. 1971, 93, 2046.

${ }^{3}$ Pool, J. A.; Bernskoetter, W. H.; Chirik, P. J. J. Am. Chem. Soc.2004, 126, 14326

${ }^{4}$ Bernskoetter, W. H.; Olmos, A. V.; Lobkovsky, E.; Chirik, P. J. Organometallics 2006, $25,1021$.

${ }^{5}$ Chirik, P. J.; Day, M. W.; Bercaw, J. E. Organometallics 1999, 18, 1873. 